\title{
DYNAMIC PERTURBATION OF A PROPAGATING CRACK
}

\author{
J. R. Willis \\ University of Cambridge, Department of Applied Mathematics and Theoretical Physics \\ Silver Street, Cambridge CB3 9EW, UK
}

\begin{abstract}
An account of recent progress in dynamic perturbation of a propagating crack is given.
\end{abstract}

\section{Outline of research developments}

This presentation summarises a solution recently obtained for the perturbation of a propagating crack. The crack propagates dynamically so that, at time $t$, it occupies the domain

$$
S_{\varepsilon}=\left\{-\infty<x_{1}-V t<\varepsilon \phi\left(x_{2}, t\right),-\infty<x_{2}<\infty, x_{3}=\varepsilon \psi\left(x_{1}, x_{2}\right)\right\}
$$

where $\varepsilon<<1$. This represents a general dynamic perturbation of a plane crack in uniform motion defined by the surface $S_{0}$, realized when $\varepsilon=0$. Maintenance of such a uniform motion would require loading to be independent of $x_{2}$ and to depend on $\left(x_{1}, t\right)$ only in the combination $X=x_{1}-$ $V t$. This special loading yields no simplification in the analysis, so the boundary value problem may just as well be solved for general loading. The basic objective is to solve the boundary value problem asymptotically, to first order in $\varepsilon$, and then to impose a fracture criterion, which restricts the functions $\phi$ and $\psi$. Rice, Ben-Zion and Kim [5] solved a problem of this type, but for the scalar wave equation, and with $\psi=0$, so that the crack remained in the plane $x_{3}=0$. In particular, they calculated the stress intensity factor in terms of the function $\phi$, and then invoked a fracture criterion in which the local toughness was specified as a function of $\left(x_{1}, x_{2}\right)$ to derive an integral equation for $\phi$. The full elastodynamic problem is technically much more difficult but it has been solved (Willis and Movchan [6], Movchan and Willis [4], and Willis and Movchan [7] ).

The technique devised by Willis and Movchan made use of an integral identity. Take $d>>$ $\varepsilon$ but still small, and take $\mathbf{u}$ to be the additional displacement generated by introducing a crack over $S_{\varepsilon}$, into an infinite domain in which the displacement and stress fields in the absence of the crack would have components $u_{i}^{A}, \sigma_{i j}^{A}$. The displacement field $\mathbf{u}$ is therefore allowed to jump across the surface $S_{\varepsilon}$, while stresses are continuous except possibly at $S_{\varepsilon}$, and satisfy the boundary conditions

$$
\sigma_{i j} n_{j}+\sigma_{i j}^{A} n_{j} \rightarrow 0
$$


as $\mathbf{x} \rightarrow S_{\varepsilon}$ from either side, $\mathbf{n}$ being the normal (with $n_{3}>0$ ) to $S_{\varepsilon}$. Denote by $\mathbf{u}(d)$ the displacement $\mathbf{u}$ evaluated on $x_{3}=d$, and let $\sigma(d)$ be the corresponding traction vector, with components $\sigma_{i 3}(d)$. These are related by the identity

$$
\mathbf{u}(d)=-\mathbf{G} * \boldsymbol{\sigma}(d)
$$

where $\mathbf{G}\left(x_{1}, x_{2}, t\right)$ represents the Green's tensor for surface loading of the half-space $x_{3}>d$ evaluated on the surface $x_{3}=d$, and $*$ represents the operation of convolution with respect to $\left(x_{1}\right.$, $x_{2}, t$ ). It follows also, by considering the half-space $x_{3}<-d$, that

$$
\mathbf{u}(-d)=\mathbf{G}^{\mathbf{T}} * \boldsymbol{\sigma}(-d)
$$

Now associate with the unperturbed configuration displacement and stress vector fields $\mathbf{U}$ and $\boldsymbol{\Sigma}$. Their values on $x_{3}= \pm d$ are similarly related as in (3) and (4). Next, note that

$$
\mathbf{U}^{\mathrm{T}}(-d)^{*} \boldsymbol{\sigma}(d)=\boldsymbol{\Sigma}^{\mathrm{T}}(-d)^{*} \mathbf{G}^{*} \boldsymbol{\sigma}(d)=-\boldsymbol{\Sigma}^{\mathrm{T}}(-d)^{*} \mathbf{u}(d)
$$

A similar identity applies with $d$ replaced by $-d$. Subtracting the two gives

$$
-\langle\mathbf{U}\rangle_{d}^{\mathrm{T}} *[\boldsymbol{\sigma}]_{d}+[\mathbf{U}]_{d}^{\mathrm{T}} *\langle\boldsymbol{\sigma}\rangle_{d}=\langle\boldsymbol{\Sigma}\rangle_{d}^{\mathrm{T}} *[\mathbf{u}]_{d}-[\mathbf{\Sigma}]_{d}^{\mathrm{T}} *\langle\mathbf{u}\rangle_{d}
$$

where

$$
\langle f\rangle_{d}=\frac{1}{2}\{f(d)+f(-d)\},[f]_{d}=f(d)-f(-d)
$$

Finally, by writing side-by-side three linearly independent solution pairs $\mathbf{U}, \boldsymbol{\Sigma}$, these may be regarded as $3 \times 3$ matrices in the fundamental identity (6) which applies for all $\varepsilon$ and $d$, subject only to the restriction $d \gg \varepsilon$. Willis and Movchan exploited the identity (6) by choosing $\mathbf{U}$ to be a weight function matrix for the unperturbed moving crack. This satisfies the equations of motion except on the plane $x_{3}=0, \Sigma$ is continuous across $x_{3}=0, \mathbf{U}$ is allowed to be discontinuous across $x_{3}=0$ when $X=x_{1}-V t>0$, and $\Sigma=0$ when $x_{3} \rightarrow \pm 0$ and $X>0$. The field $\mathbf{U}$ is not identically zero because it is chosen to have an 'unphysical' singular behaviour as $X \rightarrow 0$ with $x_{3}=0$. Specifically,

$$
[\mathbf{U}] \sim\left(\frac{2}{\pi X}\right)^{1 / 2} H(X) \delta\left(x_{2}\right) \delta(t) \mathbf{I} \quad \text { as } X \rightarrow 0
$$


This represents a dynamical generalization of the static weight functions of Bueckner [1] $]^{1}$ Here, [.] denotes the limit as $d \rightarrow 0$ of the corresponding quantity with subscript $d$. Similar notation will be deployed below for $\langle$. $\rangle$. These conditions suffice to define $\mathbf{U}$ and $\boldsymbol{\Sigma}$ uniquely. Of course, finding them is a task of substance. It follows from symmetry that Mode I (for which only [ $U_{33}$ ] $\neq 0$ ) uncouples from Modes II and III which, unlike the case of two dimensions, remain coupled. The Mode I problem can be reduced to a scalar Wiener-Hopf problem for $\left[U_{33}\right]$ and is soluble by standard means (Willis and Movchan [6]). By good fortune, the matrix Wiener-Hopf problem for the coupled Modes II and III is also soluble (Movchan and Willis [4]): this is one of very few examples of an explicit solution to such a problem.

The use of the identity (6) may be illustrated by applying it to the unperturbed crack. Then, $\varepsilon=0$ and it is possible to let $d \rightarrow 0$ immediately. Now set

$$
\boldsymbol{\sigma}=\sigma_{+}+\sigma_{-},
$$

where $\sigma_{+}=0$ for all $X<0$ (so that $\sigma_{+}=\sigma H(X)$ ), and split other functions similarly. It should be noted that the given boundary conditions imply that

$$
\langle\boldsymbol{\Sigma}\rangle=\langle\boldsymbol{\Sigma}\rangle_{-},[\mathbf{\Sigma}]=0,[\mathbf{U}]=[\mathbf{U}]_{+} \text {, and }[\mathbf{u}]=[\mathbf{u}]_{-},[\boldsymbol{\sigma}]=[\boldsymbol{\sigma}]_{-} .
$$

The identity (6), with $d=0$, now takes the form

$$
[\mathbf{U}]_{+}^{\mathrm{T}} *\langle\boldsymbol{\sigma}\rangle_{+}=-[\mathbf{U}]_{+}^{\mathrm{T}} *\langle\boldsymbol{\sigma}\rangle_{-}+\langle\mathbf{U}\rangle^{\mathrm{T}} *[\boldsymbol{\sigma}]_{-}+\langle\mathbf{\Sigma}\rangle_{-}^{\mathrm{T}} *[\mathbf{u}]_{-} .
$$

The functions $\langle\boldsymbol{\sigma}\rangle_{-},[\boldsymbol{\sigma}]_{-}$are known, from the given boundary conditions ${ }^{2}$. The function $\langle\boldsymbol{\sigma}\rangle_{+}$is not known, but it has the asymptotic form

$$
\langle\boldsymbol{\sigma}\rangle_{+} \sim \mathbf{K}\left(x_{2}, t\right) H(X) / \sqrt{2 \pi X} \text { as } \mathbf{A}^{(0)} \rightarrow 0,
$$

where the stress intensity vector $\mathbf{K}$ has components $\left(K_{\mathrm{II}} K_{\mathrm{III}}, K_{\mathrm{I}}\right)$. It is unknown, but it can be found by applying (11) as $X \rightarrow+0$. For any $X>0$, the convolution of the two ' - ' functions is zero, identically. Also, as $X \rightarrow 0$, the convolution of the two '+' functions can be evaluated from their asymptotic forms (8), (12), giving

$$
[\mathbf{U}]_{+}^{\mathrm{T}} *\langle\boldsymbol{\sigma}\rangle_{+} \sim \mathbf{K}
$$

\footnotetext{
'Bueckner's solution for the weight functions for a crack loaded asymmetrically appears to contain one or two minor slips. These are corrected, in the context of a fully general solution for the static perturbation of a stationary plane crack, in Movchan, Gao and Willis [3].

${ }^{2}$ In fact, for the conditions given, $[\sigma]=0$, but the formula also applies more generally, when the upper and lower crack faces are loaded differently.
} 
Thus, (11) gives

$$
\mathbf{K}=-[\mathbf{U}]_{+}^{\mathrm{T}} *\langle\boldsymbol{\sigma}\rangle_{-}+\langle\mathbf{U}\rangle^{\mathrm{T}} *[\boldsymbol{\sigma}]_{-},
$$

in which the convolutions are evaluated for $X=0$.

The perturbations of the stress intensity factors in the case of the perturbed crack also follow, by exploiting the fact that (6) is an identity in $\varepsilon, d$ and $X$. Their evaluation requires expansion of the identity to lowest order in $d$ and to first order in $\varepsilon$; it is also necessary to expand the unperturbed field, and the weight function, to two non-trivial terms in $X$.

The techniques required to complete the solution include Fourier transforms, complex variable theory, matched expansions and generalized functions. The final result of the perturbation theory is to represent the stress intensity factor in the form $\mathbf{K}=\mathbf{K}^{(0)}+\Delta \mathbf{K}$, with the perturbation given by

$$
\begin{aligned}
\Delta \mathbf{K}= & \varepsilon\left\{\mathbf{Q}^{\mathrm{T} *}\left(\left(\phi \mathbf{I}-\psi^{*} \boldsymbol{\Theta}\right) \mathbf{K}^{(0)}\right)-\left(\mathbf{E} \phi,_{2}+\boldsymbol{\Omega}\right) \mathbf{K}^{(0)}\right. \\
& \left.+\left(\frac{\pi}{2}\right)^{1 / 2}\left(\phi \mathbf{A}^{(0)}+\psi^{*} \mathbf{L}\right)\right\}+\left\{[\mathbf{U}]^{\mathrm{T}} *\left\langle\mathbf{P}^{(1)}\right\rangle-\langle\mathbf{U}\rangle^{\mathrm{T}} *\left[\mathbf{P}^{(1)}\right]\right\}_{(X=0)} .
\end{aligned}
$$

The terms in (15) are defined in full in Willis and Movchan [7]. The function $\mathbf{Q}$ is obtained from the asymptotic expansion of [U] as $X \rightarrow 0$. The unperturbed stress field ahead of the crack has the form

$$
\boldsymbol{\sigma} \sim \mathbf{K}^{(0)} / \sqrt{2 \pi X}-\mathbf{P}^{(0)^{*}}+\mathbf{A}^{(0)} \sqrt{X},
$$

$\psi^{*}\left(x_{2}, t\right)=\psi\left(0, x_{2}, t\right)$ and $\mathbf{P}^{(1)}$ defines the perturbation of the boundary condition, obtained by formal expansion of (2). The matrix $\mathbf{L}$ depends on $\mathbf{A}^{(0)}, \dot{\mathbf{K}}^{(0)}$ and $\mathbf{K}_{2}^{0}$, and $\mathbf{\Omega}$ depends on $\psi, 1$ and $\psi,,_{2}$; the other matrices are constants.

The solution has recently been exploited, in the special case of Mode I loading with the crack confined to the plane $x_{3}=0$, to explain the presence of Wallner lines on a crack surface (Fisher and Ramanathan [2]). The full solution will facilitate a variety of studies of crack perturbation, and crack stability; these will require additional information in the form of fracture criteria, and perhaps also further asymptotic analysis.

\section{References}

[1] Bueckner, H.F. (1987). Weight functions and fundamental solutions for the penny shaped and half-plane crack in three space. Int J. Solids Struct. 23, 57-93.

[2] Fisher, D. and Ramanathan, S. (1997). Dynamics and instabilities of planar tensile cracks in heterogeneous media. Phys. Rev. Lett. 79, 877-880. 
[3] Movchan, A.B., Gao, H. and Willis, J.R. (1998). On perturbations of plane cracks. Int. J. Solids Struct. 35, 3419-3453.

[4] Movchan, A.B. and Willis, J.R. (1995). Dynamic weight functions for a moving crack II. Shear loading. J. Mech. Phys. Solids 43, 1369-1383.

[5] Rice, J.R., Ben-Zion, Y. and Kim, K.-S. (1994). Three-dimensional perturbation solution for a dynamic planar crack moving unsteadily in a model elastic solid. J. Mech. Phys. Solids 42, 813-843.

[6] Willis, J.R. and Movchan, A.B. (1995). Dynamic weight functions for a moving crack. I. Mode I loading. J. Mech. Phys. Solids 43, 319-341.

[7] Willis, J.R. and Movchan, A.B. (1997). Three-dimensional dynamic perturbation of a propagating crack. J. Mech. Phys. Solids 45, 591-610. 
